\title{
Nanoscale
}

Check for updates

Cite this: Nanoscale, 2022, 14, 2210

\section{Noninvasive near-infrared light triggers the remote activation of thermo-responsive TRPV1 channels in neurons based on biodegradable/photothermal polymer micelles}

\author{
Wei-Hsu Chen, Taiki Onoe and Masao Kamimura (DD *
}

In this study, we developed a novel biodegradable/photothermal polymer micelle-based remote-activation method for a temperature-sensitive ion channel, namely transient receptor potential cation channel subfamily $V$ member 1 (TRPV1). Biodegradable/photothermal polymer micelles containing indocyanine green (ICG-micelles) were prepared using a simple one-pod mixing method. The obtained ICGmicelles showed biocompatibility and biodegradability. Furthermore, under tissue-penetrable near-infrared (NIR) laser irradiation, the ICG-micelles exhibited excellent photothermal effects and NIR emission. Moreover, NIR light-induced remote activation of neurons was successfully performed. ICG-micelles loaded with anti-TRPV1 antibodies effectively bound TRPV1 on cell membranes, and accelerated $\mathrm{Ca}^{2+}$ ion influx into neuronal cells was induced under NIR irradiation. Based on these findings, it is anticipated that the ICG-micelles can serve as a novel noninvasive remote-activation tool for neuronal cells.
Received 2nd November 2021, Accepted 7th January 2022

DOI: $10.1039 / \mathrm{d} 1 \mathrm{nr} 07242 \mathrm{k}$

rsc.li/nanoscale membranes using external stimuli and stimuli-responsive nanomaterials. Under external stimuli (such as a magnetic field, ${ }^{4-8}$ ultrasound waves, ${ }^{9,10}$ or light irradiation, ${ }^{11,12}$ mechano- or temperature-sensitive ion channels are activated and an ion efflux and/or influx is promoted, resulting in the activation of cellular functions. In addition, these manipulation techniques have great potential for novel therapeutic applications for intractable diseases, ${ }^{13,14}$ such as neurodegenerative diseases ${ }^{15-19}$ and cancer..$^{20-23}$

In particular, photo-responsive nanomaterials are the most promising candidates for the remote activation of cellular ion channels. $^{2}$ It is well known that photo-irradiation-based techniques for cellular manipulation or observation are superior to other approaches, given their spatiotemporal controllability. Thus, fluorescence microscopy and various photo-responsive reagents, including fluorescent markers, are commonly used in biological research. Regarding the cell-manipulation method, optogenetics is a paradigm-shifting technique based on photo-irradiation. ${ }^{24-26}$ Optogenetics is a method for manipulating cell functions using genetically encoded light-sensitive ion channels and light irradiation. This technique can control a neuron's activity using light irradiation and thus has great potential for cranial nerve research. However, most current optogenetics approaches are difficult to use in deep tissues because of limited light penetration. Although most optogenetics methods use short-wavelength blue or green light-sensitive ion channels, these short wavelength lights permeate poorly into the body due to strong light absorption, as well as 
scattering off of various biomolecules and water in the body. To address this issue, tissue-penetrable near-infrared (NIR) light-induced optogenetics methods, based on upconversion nanoparticles (UCNPs), have been proposed. ${ }^{27-29}$

The NIR wavelength region (700-1700 nm) is well known as a tissue-penetrable "biological window" region. ${ }^{30}$ Because of the low absorption or scattering of biomolecules and water in this region, NIR light can penetrate deep tissues. UCNPs can be manipulated to emit short-wavelength visible light under NIR excitation by using a stepwise excitation scheme. Thus, UCNP-based optogenetics can control ion channels responsive to blue or green light under NIR light irradiation and can be used for deep tissue in vivo manipulation. However, it is difficult to use optogenetics approaches in human clinical applications because genetic engineering is required to express light-sensitive ion channels in the cells of interest.

More recently, temperature-sensitive ion channels that naturally exist in the body have received much attention as targets of remote activation. For example, transient receptor potential cation channel subfamily V member 1 (TRPV1) is one of the most well-known temperature-responsive ion channels expressed on the cell membrane. ${ }^{31}$ TRPV1 is well known as the capsaicin receptor; this receptor is also opened by high temperatures $\left(>43{ }^{\circ} \mathrm{C}\right)$, which accelerates the influx of cations. In addition, because TRPV1 is widely expressed throughout the nervous system, it is a potential target for neuronal cell activation. Several researchers have proposed the remote activation of the TRPV1 channel by eliciting the "photothermal effect" with various nanomaterials, such as carbon nanomaterials, ${ }^{32}$ gold nanorods, ${ }^{17,33,34}$ semiconductor quantum dots, ${ }^{22,23}$ and synthesized semiconductor polymer dots. ${ }^{18,20}$ It is well known that these nanomaterials generate an increase in the temperature under NIR photo-irradiation. This phenomenon is known as the "photothermal effect", and it is well established for use in cancer photothermal therapy (PTT). ${ }^{35}$ However, the synthesis of these nanomaterials requires techniques that can be adapted to large-scale production, considering that the price of commercially available materials may be prohibitive. Thus, it is difficult to popularise these materialbased, cell-activation techniques in normal laboratories. In addition, most of these NIR light-responsive nanomaterials are inorganic or metal nanomaterials. Thus, these materials are difficult to use in human clinical purposes, because of their toxicity and problems in disposing of them after use.

To address these issues, it is expected that a low-cost and safe material-based photothermal activation method for TRPV1 will be developed. For example, indocyanine green $(\mathrm{ICG})^{36}$ is a low-cost and commercially available organic dye. ICG has already been approved by the Food and Drug Administration in the US, and the low molecular weight dye can be cleared from the blood through the kidneys. Furthermore, ICG has shown excellent photothermal effects under NIR light irradiation. For example, ICG-based cancer PTT under tissue-penetrable NIR light irradiation has been performed successfully. ${ }^{37,38}$ In addition, ICG also shows NIR emission under NIR excitation; thus, it can also be used as a fluorescence bioimaging probe. ${ }^{39,40}$ Therefore, ICG is a promising molecule for theranostic applications. Thus, ICG is a potentially safe candidate for photothermal TRPV1 activation. However, because ICG possesses cyanine structures, it is hardly soluble in water, which makes it difficult to use in an aqueous environment.

Amphiphilic block copolymer-based polymer micelles are widely used as nanocarriers to deliver hydrophobic low-molecular weight molecules, such as drugs or dyes in the body. ${ }^{41,42}$ In these previous studies, hydrophobic molecules were loaded into the stable hydrophobic cores of polymer micelles, and the hydrophilic shell blocked the attack of external biomolecules and improved the dispersion stability of polymer micelles under physiological conditions. Thus, encapsulation of hydrophobic dyes is a promising method for successfully using dyes in aqueous environments.

Therefore, we first developed ICG dye-loaded biodegradable polymer micelles based on ICG and a biodegradable and amphiphilic block copolymer, poly(ethylene glycol) (PEG)block-poly( $\varepsilon$-caprolactone) (PCL) (PEG- $b$-PCL), which were used to photothermally activate the TRPV1 ion channel of neurons (Fig. 1). PEG- $b$-PCL is formed from a hydrophilic PEG segment and a hydrophobic PCL segment, and thus can easily form a stable polymeric micelle structure with a hydrophobic lowmolecular weight dye, ICG, in water via a simple "one-pod" scheme. In addition, because the PCL segment contains biodegradable ester bonds, it is degraded by hydrolytic cleavage in the body. ${ }^{43}$ Therefore, PEG- $b$-PCL-based polymer micelles are widely used as nanocarriers for hydrophobic anticancer drugs and fluorescent dyes. ${ }^{44,45}$ Thus, the ICG and PEG- $b$-PCL based polymer micelles possess biocompatibility and biodegradability, and they can be used as a novel and safe photothermal activation tool for TRPV1. In addition, PEG- $b$-PCL with various molecular weights is commercially available from various chemical companies. Importantly, the photothermal nanomaterials used for TRPV1 activation in this study were produced using only commercially available reagents and can be easily prepared without special synthesis techniques.

\section{Materials and methods}

\section{Materials}

PEG- $b$-PCL $\left(M_{\mathrm{n}}: 5000-4000\right)$ and PEG- $b$-PCL possessing an amine group at the $\alpha$ end of the PEG chain ( $\mathrm{NH}_{2}$-PEG- $b$-PCL) $\left(M_{\mathrm{n}}: 5000-4000\right)$ were purchased from Polymer Source (Montreal, QC, Canada). ICG, Nile red (NR), and 1-(3-dimethylaminopropyl)3-ethylcarbodiimide hydrochloride (EDC) were purchased from Tokyo Chemical Industry (Tokyo, Japan). Acetonitrile, dimethyl sulfoxide (DMSO), $N$-hydroxysuccinimide (NHS), capsaicin, Hank's buffer with HEPES (HHBS), and penicillin-streptomycin were purchased from FUJIFILM Wako Pure Chemical Corporation (Osaka, Japan). Pluronic® F-68 was purchased from SigmaAldrich (St Louis, MO, USA). Fetal bovine serum (FBS) was purchased from Biowest (Nuaille, France). Dulbecco's modified Eagle's medium (DMEM), the Micro BCA Assay Kit, and 


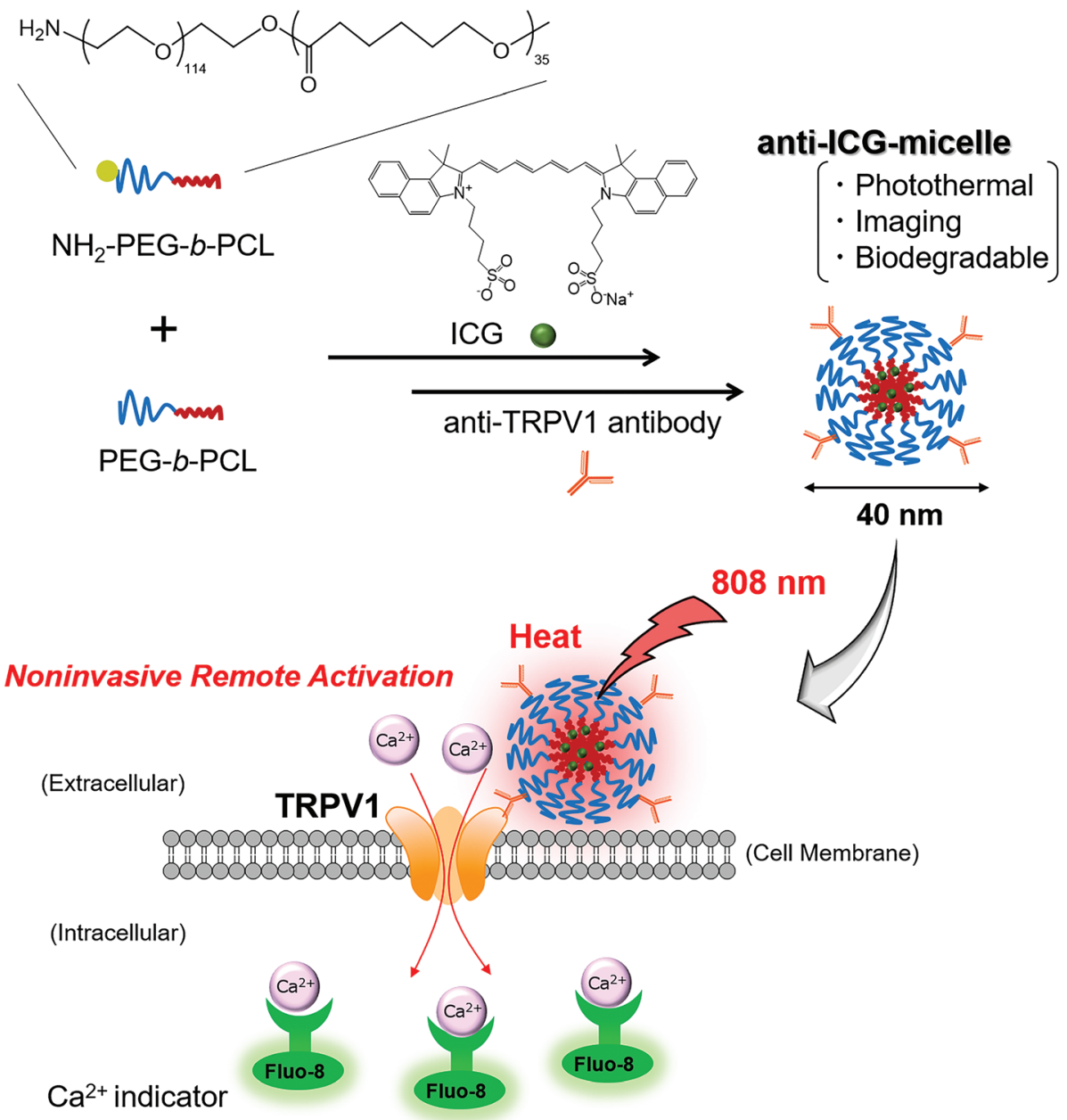

Accelerated $\mathrm{Ca}^{2+}$ Influx into Neuronal Cells

Fig. 1 Schematic illustration of the NIR light-induced remote photoactivation of TRPV1 on a neuron.

CellMask $^{\mathrm{TM}}$ Green Plasma Membrane Stain were purchased from Thermo Scientific (Waltham, MA, USA). The Cell Counting Kit-8 containing a water-soluble tetrazolium (WST) assay reagent was purchased from Dojindo Laboratories (Kumamoto, Japan). An anti-TRPV1 antibody was purchased from Proteintech Group (IL, USA). A Fluo- $8 \AA \mathrm{Ca}^{2+}$ indicator was purchased from AAT Bioquest (CA, USA). All reagents were used without further purification.

\section{Preparation of ICG-loaded polymer micelles (ICG-micelles)}

ICG-micelles were prepared as follows. PEG- $b$-PCL ( $4 \mathrm{mg}$ ) and ICG $(20 \mu \mathrm{g})$ were dissolved in $1 \mathrm{~mL}$ of acetonitrile in a vial, followed by the addition of $8 \mathrm{~mL}$ of ultrapure water. Then, acetonitrile was allowed to evaporate from the aqueous ICG-micelle dispersion while stirring it for $12 \mathrm{~h}$ at $25{ }^{\circ} \mathrm{C}$. The obtained ICG-micelles were purified by centrifugation four times through a filter (MWCO: $10 \mathrm{kDa}, 3500 \mathrm{~g}, 15 \mathrm{~min}$ ) and then dispersed in ultrapure water.

\section{Preparation of anti-ICG-micelles and anti-NR-micelles}

Anti-TRPV1 antibody-labelled micelles loaded with ICG (antiICG-micelles) and anti-TRPV1 antibody-labelled micelles loaded with NR (anti-NR-micelles) were prepared using a similar method. The anti-ICG-micelles were prepared as follows: PEG- $b$-PCL ( $3 \mathrm{mg}$ ), $\mathrm{NH}_{2}$-PEG- $b$-PCL $(1 \mathrm{mg})$, and ICG $(20 \mu \mathrm{g})$ were dissolved in $1 \mathrm{~mL}$ of acetonitrile in a vial, followed by the addition of $8 \mathrm{~mL}$ of ultrapure water. Then, acetonitrile was allowed to evaporate from the aqueous dispersion of ICGmicelles possessing $\mathrm{NH}_{2}$ groups while stirring for $12 \mathrm{~h}$ at $25{ }^{\circ} \mathrm{C}$. The obtained ICG-micelles (possessing $\mathrm{NH}_{2}$ groups) were purified four times by centrifugation through a filter (MWCO: $10 \mathrm{kDa}, 3500 \mathrm{~g}, 15 \mathrm{~min}$ ), and then the obtained micelles were dispersed in $2 \mathrm{~mL}$ of phosphate buffer (PB; $10 \mathrm{mM}, \mathrm{pH}$ 7.4). Subsequently, $100 \mu \mathrm{L}$ of EDC solution (40 $\mathrm{mM}$ in MES buffer, $\mathrm{pH}$ 6.0) and $100 \mu \mathrm{L}$ of NHS solution (40 mM in MES buffer, pH 6.0) were mixed, after which $2 \mu \mathrm{g}$ of anti-TRPV1 antibody was added to the mixture, followed by gentle shaking for $20 \mathrm{~min}$. Next, $2 \mathrm{~mL}$ of micelle dispersion was added to the antibody solution, and the resulting mixture was incubated for $12 \mathrm{~h}$. The obtained anti-ICG-micelles were purified four times by centrifugation through a filter (MWCO: $300 \mathrm{kDa}, 3500 \mathrm{~g}, 15 \mathrm{~min}$ ), and the obtained micelles were dispersed in ultrapure water. Antibody immobilisation on the 
micelles was evaluated using the standard Micro BCA protein assay method. After the stoichiometric components of the antiICG-micelle aqueous solution and the Micro BCA reagent solution were mixed and stirred for $1 \mathrm{~h}$ at $60^{\circ} \mathrm{C}$, the absorbance of the solution at $562 \mathrm{~nm}$ was measured using an ultravioletvisible (UV-VIS) light spectrophotometer (V-650, JASCO, Tokyo, Japan). Anti-NR-micelles were prepared using the same procedure, except that NR $(2.5 \mu \mathrm{g})$ was used as the encapsulate dye.

\section{Characterization of the micelle samples}

The particle size of the micelle samples was measured by dynamic light scattering (DLS) using an ELSZ-2000 ZS instrument (Otsuka Electronics, Osaka, Japan). The absorption spectra of the micelle samples were recorded using a UV-VIS spectrophotometer (V-650, JASCO). The loading amounts of dyes in the micelles were calculated from the absorbance of the samples. The micelle samples were dissolved in acetonitrile and the absorption spectrum was recorded. The loading amounts of ICG in the ICG-micelles and NR in the NR-micelles were determined by measuring the absorbance at $785 \mathrm{~nm}$ and $535 \mathrm{~nm}$, respectively. The emission spectra of the micelle samples were recorded using a spectrometer (RF-6000, Shimadzu, Kyoto, Japan). The stabilities of the encapsulated dyes in the micelle samples were evaluated by measuring the release rates of the dyes from the micelles. The ICG-release properties of the ICG-micelles were evaluated as follows. The release of ICG from the micelle core was examined in phosphate buffered saline (PBS; $10 \mathrm{mM}, \mathrm{pH} 7.4,150 \mathrm{mM} \mathrm{NaCl}$ ) at $25{ }^{\circ} \mathrm{C}$ by dialysis with a membrane (MWCO: $\left.3500 \mathrm{Da}\right)$. At defined time intervals (0-24 h), $1 \mathrm{~mL}$ samples of dialysate solution were withdrawn and replaced with an equal volume of fresh media. The ICG concentrations in the dialysate samples were determined spectrophotometrically by measuring the absorbance at $785 \mathrm{~nm}$. NR dye release from the NR-micelles was evaluated using the same procedure, except that the NR concentrations in the dialysate samples were determined spectrophotometrically by measuring the absorbance at $535 \mathrm{~nm}$.

\section{Evaluation of the hydrolytic degradation of ICG-micelles}

Hydrolytic degradation of ICG-micelles was evaluated under acidic conditions. The ICG-micelles were dispersed in $\mathrm{HCl}$ solution (0-1 M) and stirred for $48 \mathrm{~h}$ at $25{ }^{\circ} \mathrm{C}$. ICG-micelle degradation was evaluated using DLS measurements.

\section{Evaluation of the photothermal effect of ICG-micelles}

The ICG-micelles ( $50 \mu \mathrm{g} \mathrm{mL}^{-1}$ on ICG) were dispersed in ultrapure water and poured into cuvettes. The photothermal effect of the ICG-micelles was evaluated using a thermometer (SN-3000, Netsuken, Tokyo, Japan) under $808 \mathrm{~nm}$ laser (1 W $\mathrm{cm}^{-2}$ ) irradiation (BWF2 laser; B\&W Tek, DE, USA). During the temperature measurements, the sample solutions were stirred using a micromagnetic stirrer bar.

\section{Cell culture}

Mouse neuroblastoma/rat dorsal root ganglion neuron hybrid ND7/23 cells were obtained from the European Collection of
Authenticated Cell Cultures (Salisbury, UK). ND7/23 cells were cultured in DMEM containing 10\% FBS and 1\% penicillinstreptomycin at $37^{\circ} \mathrm{C}$ in a humidified atmosphere containing $5 \% \mathrm{CO}_{2}$.

\section{Cell cytotoxicity}

The cellular cytotoxicity of the micelle samples was evaluated using the WST assay. The cytotoxicity of ICG-micelles was evaluated as follows: ND7/23 cells were seeded in a 96-well plate at $1 \times 10^{4}$ cells per well. After incubation for $24 \mathrm{~h}$, followed by washing with PBS, ICG-micelles were added to each well at various concentrations $\left(0.01-50 \mu \mathrm{g} \mathrm{mL}^{-1}\right.$ on ICG). After incubation for $24 \mathrm{~h}$, followed by washing with PBS, the cell viability was evaluated using the WST assay. The absorption value at $450 \mathrm{~nm}$ was measured using a microplate reader (Multiskan FC, Thermo Scientific). The cytotoxicity of anti-NR-micelles was evaluated using the same procedure, except that the cells were exposed to anti-NR-micelles at various concentrations (0.025-2.5 $\mu \mathrm{g} \mathrm{mL} \mathrm{m}^{-1}$ on NR).

\section{Cell cytotoxicity of the photothermal effect under NIR irradiation}

The photo- and thermal toxicities of the photothermal effect of ICG-micelles were assessed using a method similar to that described above for studying the cellular cytotoxicity of the micelle samples. ND7/23 cells were seeded in a 96-well plate at $1 \times 10^{4}$ cells per well. After incubation for $24 \mathrm{~h}$, the cells were exposed to ICG-micelles ( $50 \mu \mathrm{g} \mathrm{mL} \mathrm{m}^{-1}$ on ICG) for $3 \mathrm{~h}$, washed with PBS, and then irradiated with an $808 \mathrm{~nm}$ laser $\left(1 \mathrm{~W} \mathrm{~cm}^{-2}\right)$ for $3 \mathrm{~min}$ at $25{ }^{\circ} \mathrm{C}$ in a normal atmosphere. After washing the cells with PBS, the viability was evaluated by performing WST assays and measuring the absorbance at $450 \mathrm{~nm}$.

\section{Fluorescence imaging of anti-NR micelle labelling on the cell membrane}

Cell membrane labelling with anti-NR-micelles was characterised by fluorescence microscopy using an IX81 microscope (Olympus, Tokyo, Japan). ND7/23 cells were plated in live cell chambers with $1 \times 10^{5}$ cells and allowed to adhere for $24 \mathrm{~h}$. Then, the cells were exposed to anti-NR-micelles for $30 \mathrm{~min}$ or $3 \mathrm{~h}$. In parallel, the cells were incubated for $30 \mathrm{~min}$ with CellMask $^{\mathrm{TM}}$ Green Plasma Membrane Stain, a cell membrane marker. The concentration of NR in the medium was $2.5 \mu \mathrm{g}$ $\mathrm{mL}^{-1}$. Finally, the cells were washed three times with PBS and maintained in complete media for fluorescence microscopy imaging.

\section{Photothermal manipulation of ND7/23 cells}

ND7/23 cells were placed in live cell chambers with $1 \times$ $10^{5}$ cells and allowed to adhere for $24 \mathrm{~h}$. Then, the cells were exposed to anti-ICG-micelles $\left(50 \mu \mathrm{g} \mathrm{mL}^{-1}\right.$ on ICG) for 3 h. Then, the cells were washed three times with PBS. Next, the cells were incubated for $1 \mathrm{~h}$ with $1 \mathrm{~mL}$ of DMEM containing Fluo-8® $\left(2 \mu \mathrm{M}\right.$ Fluo-8®, 0.02\% Pluronic ${ }^{\circledR}$ F-68, $0.004 \%$ DMSO, $99.976 \%$ HHBS), a marker of free $\mathrm{Ca}^{2+}$. 
Finally, the cells were washed three times with PBS and maintained in complete media for the photothermal experiments. The cells were irradiated with an $808 \mathrm{~nm}$ laser $\left(1 \mathrm{~W} \mathrm{~cm}{ }^{-2}\right.$ ) for $3 \mathrm{~min}$ at $25{ }^{\circ} \mathrm{C}$ in a normal atmosphere, and fluorescence images were obtained using a fluorescence microscope (IX81, Olympus). Cell manipulation using capsaicin was also evaluated with a similar procedure, except that the cells were incubated in $1 \mathrm{~mL}$ of DMEM containing capsaicin (100 $\mu \mathrm{M}, 1 \%$ DMSO, 99\% DMEM). The average pixel intensity of the observed images was determined using ImageJ software (National Institute of Health, MD, US).

\section{Results and discussion}

In this study, ICG-micelles were formed from hydrophobic ICG and an amphiphilic block copolymer, PEG- $b$-PCL, via hydrophobic interactions. The hydrodynamic particle size of the prepared ICG-micelles was characterised using DLS. The hydrodynamic particle size and polydispersity index of the ICG-micelles were $\sim 35 \mathrm{~nm}$ and 0.175 , respectively (Fig. 2(a)). Therefore, the ICG-micelles were successfully prepared.

Next, the optical characteristics of the ICG-micelles were evaluated (Fig. 2(b)). Fig. 2(b) shows the absorption and emis-
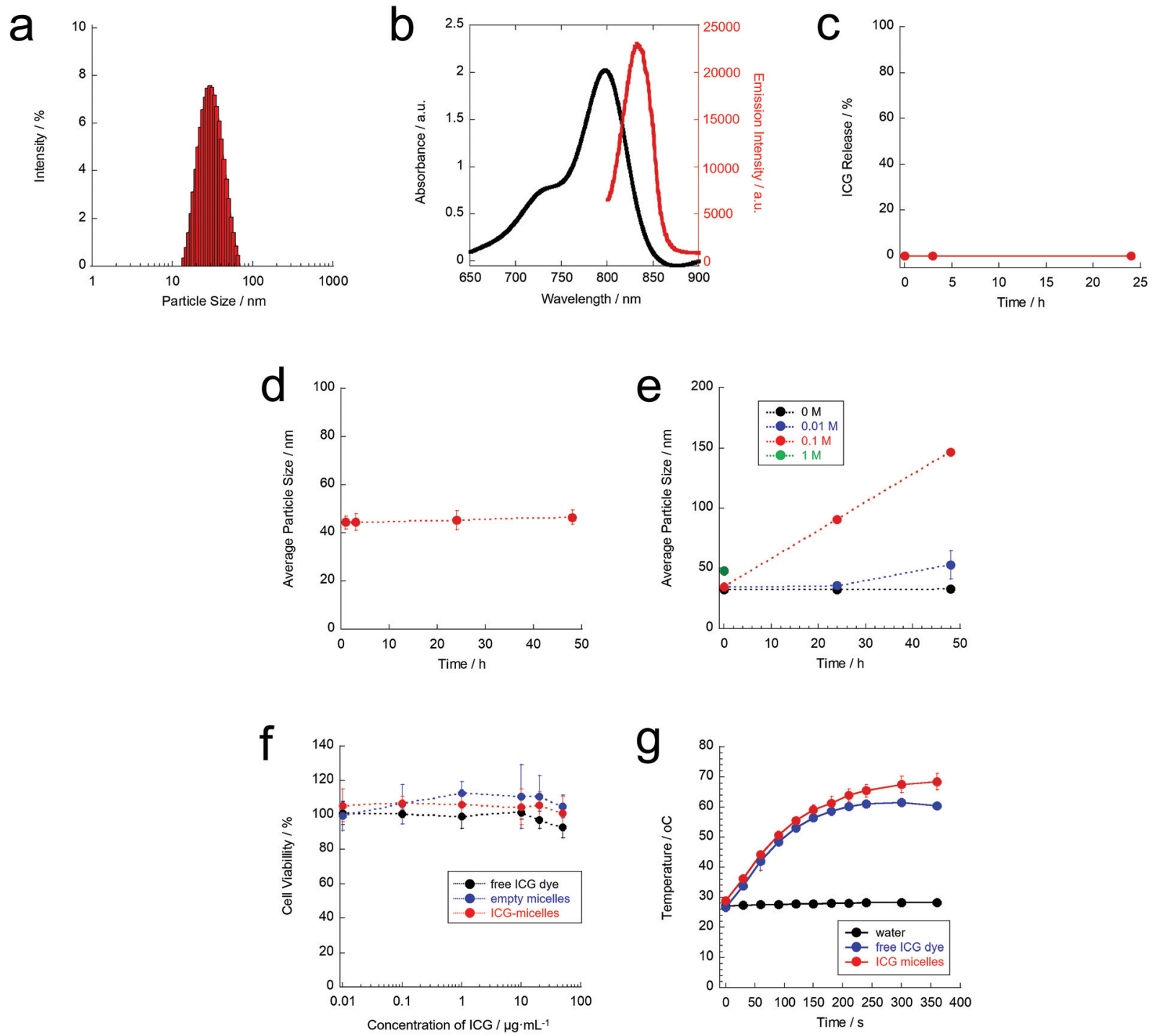

Fig. 2 Characterization of the ICG-micelles. (a) Particle size distribution of the ICG-micelles in ultrapure water, as measured by DLS. (b) Absorption (black line) and emission (red line) spectra of the ICG-micelles in ultrapure water. Excitation was performed at $790 \mathrm{~nm}$. (c) Time-dependent rate of ICG release from the ICG-micelles in PBS. The data are presented as the mean \pm SD $(n=3)$. (d) Time dependence of the average particle size of the ICG-micelles in PBS, as measured by DLS. The data are presented as the mean \pm SD $(n=3)$. (e) Hydrolysis of the ICG-micelles in the presence of different $\mathrm{HCl}$ concentrations. The data are presented as the mean $\pm \mathrm{SD}(n=3)$. (f) Cytotoxicity of the ICG-micelles against ND7/23 cells. Cytotoxicity was evaluated by performing WST assays. The data are presented as the mean \pm SD $(n=4)$. $(g) 808 \mathrm{~nm}$ NIR-induced photothermal effect of the ICG-micelles in ultrapure water. The data are presented as the mean $\pm \operatorname{SD}(n=3)$. 
sion spectra of the ICG-micelles in pure water. A distinct absorption spectrum for the ICG-micelles was obtained. This result indicates that ICG was successfully loaded into the ICGmicelles. The amount of dye loading was calculated from the absorbance values. Our results showed that the loading amount of the dye in the ICG-micelles was $0.4 \mathrm{wt} \%$. In addition, the ICG-micelles showed a strong NIR emission (830 $\mathrm{nm}$ ) under NIR excitation (790 $\mathrm{nm}$ ) in an aqueous environment. Thus, the prepared ICG-micelles also have the potential for NIR fluorescence imaging applications.
Furthermore, the stability of the ICG-micelles was evaluated in PBS. No ICG leakage was confirmed for $24 \mathrm{~h}$ (Fig. 2(c)), indicating that ICG loading in the core of ICG-micelles was quite stable. Moreover, the average particle size of the ICG-micelles remained constant (ca. 40-50 nm) for at least $48 \mathrm{~h}$ (Fig. 2(d)). These results clearly showed that the obtained ICG-micelles were stable in PBS and could be used for biological experiments without dye leakage and particle aggregation.

The biodegradability of nanomaterials after use is required for the safe use of nanomaterials in the body. Because the PCL
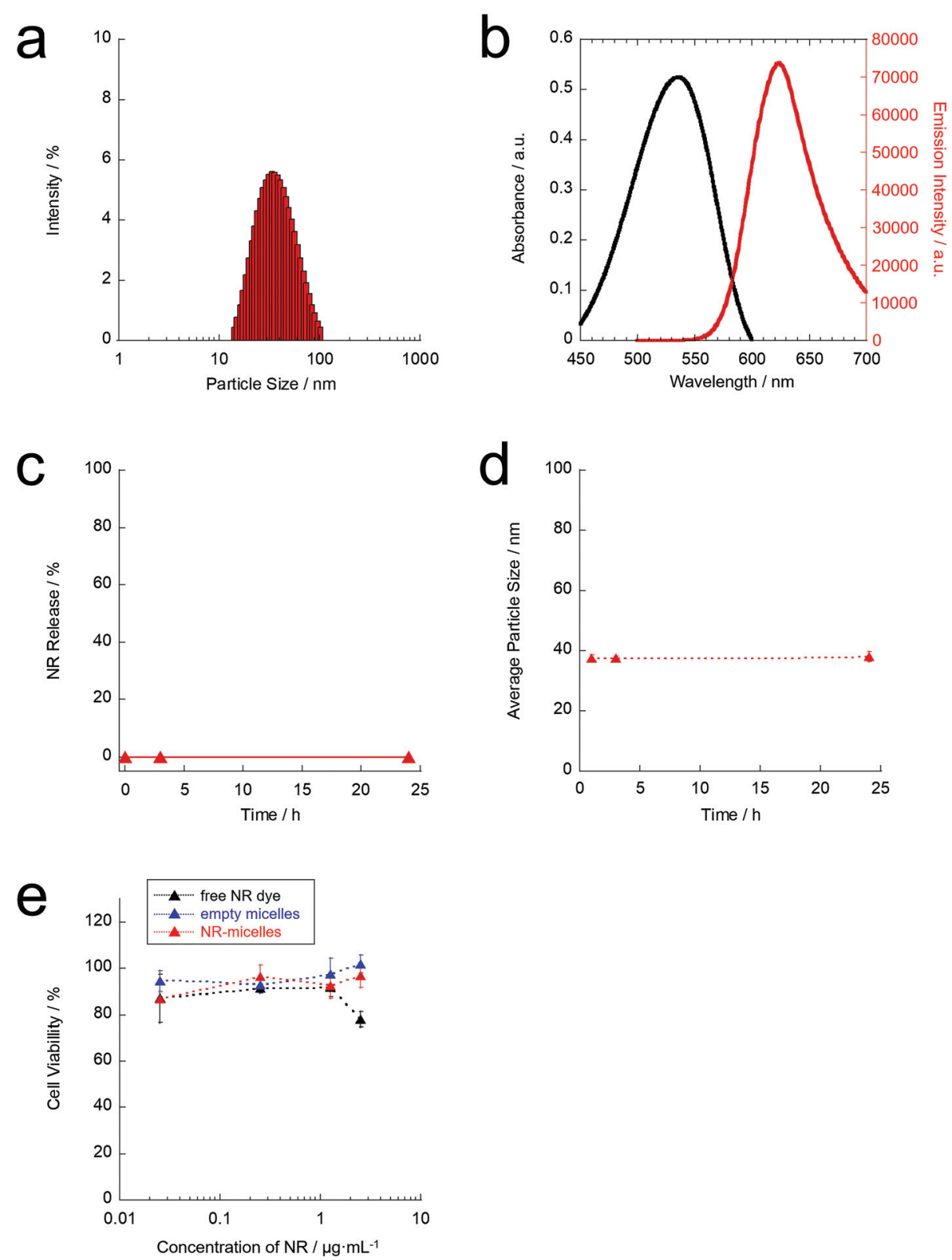

Fig. 3 Characterization of the anti-NR-micelles. (a) Particle-size distribution of the anti-NR-micelles in ultrapure water, as measured by DLS. (b) Absorption (black line) and emission (red line) spectra of the anti-NR-micelles in pure water. Excitation was performed at $540 \mathrm{~nm}$. (c) Time-dependent rate of NR dye release from anti-NR-micelles in PBS. The data are presented as the mean \pm SD $(n=3)$. (d) Time dependence of the average particle size of the anti-NR-micelles in PBS, as measured by DLS. The data are presented as the mean \pm SD ( $n=3)$. (e) Cytotoxicity of the anti-NRmicelles against ND7/23 cells. Cytotoxicity was evaluated by performing WST assays. The data are presented as the mean \pm SD $(n=4)$. 
segment of PEG- $b$-PCL possesses ester bonds, it can be degraded by hydrolytic cleavage in an aqueous environment. However, hydrolytic cleavage under neutral $\mathrm{pH}$ conditions such as physiological $\mathrm{pH}$ ( $\mathrm{pH}$ 7.4) occurs slowly because of the mild effect of hydrolysis. Therefore, accelerated hydrolytic degradation of the ICG-micelles was evaluated (Fig. 2(e)). The ICG-micelles were dispersed in aqueous hydrochloric acid $(\mathrm{HCl})$ solutions, and the particle size of ICG-micelles was measured. Our results showed that the average hydrodynamic particle size of the ICG-micelles was almost constant in ultrapure water $(0 \mathrm{M} \mathrm{HCl})$ over the course of $48 \mathrm{~h}$. However, under acidic conditions, the average hydrodynamic particle size of the ICGmicelles clearly increased with increasing $\mathrm{HCl}$ concentrations ( 0.01 and $0.1 \mathrm{M} \mathrm{HCl}$ ). These changes were probably caused by hydrolysis of the ester bonds of the PCL segment. It causes agglomeration of the micelles and/or hydrophobic ICG dyes. Furthermore, at a high $\mathrm{HCl}$ concentration $(1.0 \mathrm{M} \mathrm{HCl})$, the particle size of the ICG-micelles could be measured just after dispersing in a solution $(0 \mathrm{~h})$ with a slight increase in the particle size. However, it could not be measured after $24 \mathrm{~h}$ and $48 \mathrm{~h}$. A plausible explanation for this finding is that the ICG-micelles were degraded immediately under highly acidic conditions. These results suggest that the ICG-micelles are biodegradable.

Furthermore, the low toxicity of nanomaterials is important for successful cell manipulation. Thus, the cytotoxicity of the ICG-micelles was evaluated using a standard WST assay (Fig. 2(f)). Our results showed that the free ICG dye alone did not show any obvious cytotoxicity against ND7/23 neuron cells.
Furthermore, empty micelles did not show cytotoxicity. In addition, the ICG-micelles did not affect cell viability, even at high concentrations $\left(\leq 50 \mu \mathrm{g} \mathrm{mL}^{-1}\right.$ on ICG). Therefore, the ICG-micelles elicited minimal cytotoxicity and can be used as safe materials for cellular studies.

Next, we evaluated the photothermal properties of ICGmicelles under $808 \mathrm{~nm}$ NIR laser irradiation (Fig. 2(g)). The results showed that laser irradiation at $808 \mathrm{~nm}$ did not cause any temperature increase in water. It is well known that water does not absorb $808 \mathrm{~nm}$ NIR light, which explains why the temperature of water did not increase. ${ }^{30,46}$ In addition, the absorption and scattering of biomolecules in the body are also minimised in this wavelength region. Thus, $808 \mathrm{~nm}$ is the most tissue-penetrable wavelength in the NIR region (700-1800 nm), ${ }^{30}$ and is suitable not only for fluorescence imaging, but also light-induced manipulation techniques. In contrast, free ICG molecules showed a time-dependent increase in the temperature after laser irradiation. The temperature of the sample increased to approximately $60{ }^{\circ} \mathrm{C}$ at $4 \mathrm{~min}$ after laser irradiation. Thus, ICG possesses sufficient photothermal activity under $808 \mathrm{~nm}$ NIR laser irradiation. Furthermore, the ICG-micelles also exhibited ideal photothermal effects in aqueous environments. The temperatureincrease profile of the ICG-micelles under $808 \mathrm{~nm}$ laser irradiation was almost the same as that of free ICG molecules, and the temperature of the ICG-micelle dispersion reached $43{ }^{\circ} \mathrm{C}$ (i.e. the activation temperature of TRPV1) at 1 min after laser irradiation. Thus, the ICG-micelles can be used as photo-

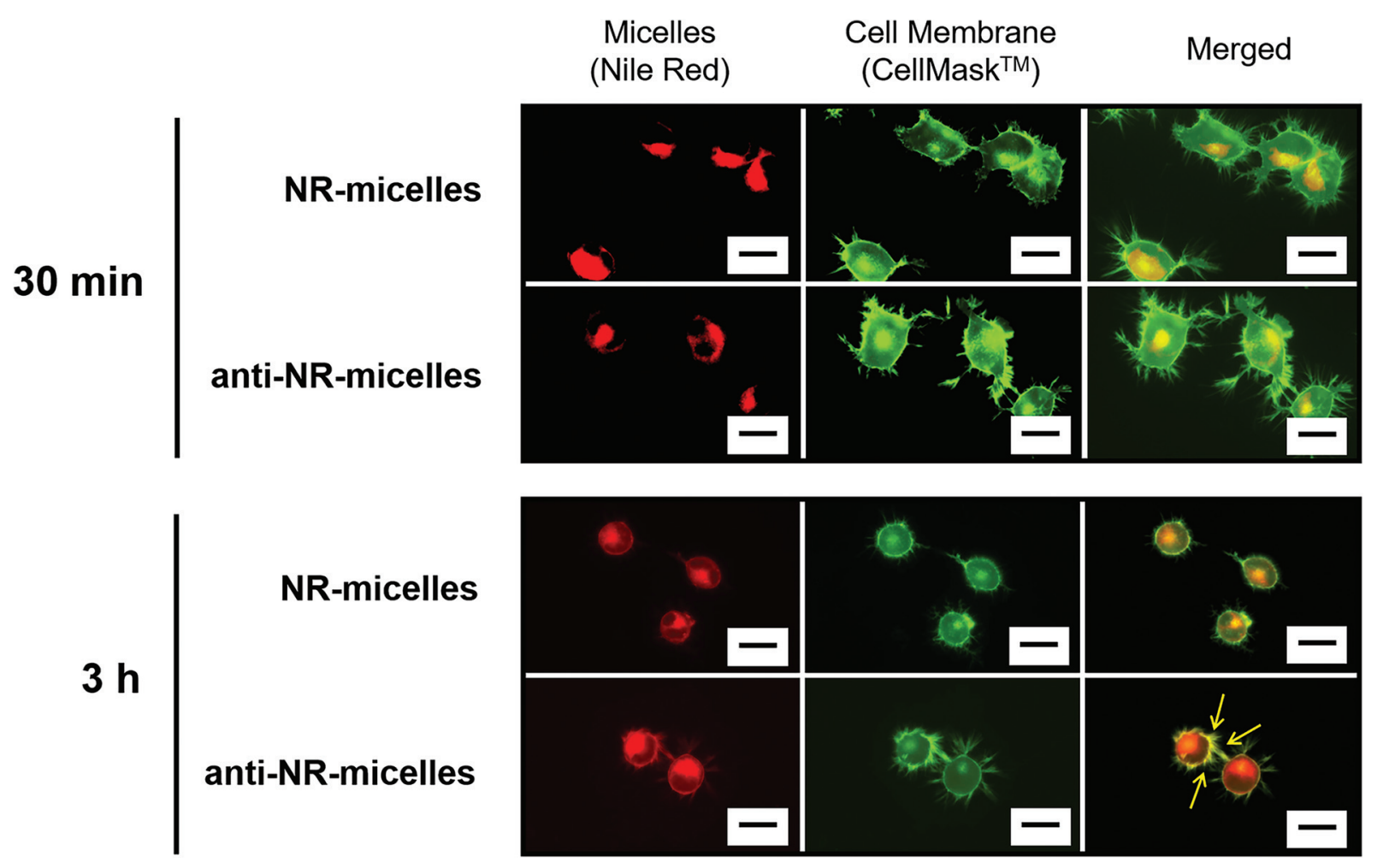

Fig. 4 Fluorescence microscopy images of ND7/23 cells incubated with NR-micelles or anti-NR-micelles. Scale bar $=20 \mu \mathrm{m}$. 
thermal transducers. Based on these characteristics, we anticipate that the ICG-micelles can serve as stable, safe, and effective photothermal materials, and thus they can be successfully used as a tool for manipulating TRPV1.

Ideal manipulation of the TRPV1 channel also requires the targeted activation of TRPV1 on the cell membrane. Therefore, the surfaces of the micelles were labelled with an anti-TRPV1 antibody to enable targeted delivery to the TRPV1 channels on the cell membrane. Fluorescence microscopy observation was carried out to study micelle localisation on the target cells. However, because the emission wavelength of ICG is in the NIR region (830 $\mathrm{nm}$ emission under $780 \mathrm{~nm}$ excitation; Fig. 2(b)), the emission of ICG-micelles is difficult to observe using normal fluorescence microscopy. Thus, we prepared micelles loaded with a fluorescent dye that emits in the visible wavelength region for fluorescence microscopy observations and micelle tracking in the cells. To this end, NR dye loaded micelles, NR-micelles, were prepared using almost the same procedure used for preparing ICG-micelles, as described above. Then, an anti-TRPV1 antibody was incorporated into the surface of the micelles (anti-NR-micelles).

The hydrodynamic particle size of the obtained anti-NRmicelles was characterised by DLS. The hydrodynamic particle size and polydispersity index of the anti-NR-micelles were approximately $40 \mathrm{~nm}$ and 0.222 , respectively (Fig. 3(a)). Immobilisation of the anti-TRPV1 antibodies on the micelle surfaces was confirmed by performing standard Micro BCA protein assays. Furthermore, the optical properties of the antiNR-micelles were investigated. Fig. 3(b) shows the absorption and emission spectra of the anti-NR-micelles in pure water. These results indicate that the anti-NR-micelles could show visible emission in an aqueous environment. The overall NRloading efficiency of the anti-NR-micelles was calculated to be $0.05 \mathrm{wt} \%$ (expressed as the mass of incorporated NR relative to the mass of the original NR and PEG- $b$-PCL mixture). Moreover, the anti-NR-micelles showed visible emission at $640 \mathrm{~nm}$ following excitation at $540 \mathrm{~nm}$ in an aqueous environment. Therefore, the emission of the anti-NR-micelles can be observed using normal fluorescence microscopy.

Furthermore, the stability of the anti-NR-micelles was also characterised in PBS. The anti-NR-micelles did not show NR leakage over the course of $24 \mathrm{~h}$ (Fig. 3(c)), and the average particle size of the anti-NR-micelles was almost constant (approximately 35-40 nm) for at least $24 \mathrm{~h}$ (Fig. 3(d)). These results suggest that the anti-NR-micelles are stable in PBS. Additionally, the cytotoxicity of the anti-NR-micelles was evaluated by performing WST assays (Fig. 3(e)). As shown in Fig. 3(e), the free NR dye and empty micelles did not exhibit obvious cytotoxicity against ND7/23 neuron cells. Furthermore, the anti-NR-micelles did not show cytotoxicity even at high dye concentrations ( $\leq 1 \mu \mathrm{g} \mathrm{mL} \mathrm{mL}^{-1}$ on NR). Therefore, the anti-NRmicelles were considered to be safe materials that could be used for further experiments.

Adsorption of anti-NR-micelles to TRPV1 on the cell membrane was observed in TRPV1-positive (+) ND7/23 cells. Fig. 4 shows the fluorescence microscopy images of ND7/23 cells incubated with NR-micelles (without antibody conjugation or anti-NR-micelles). After $30 \mathrm{~min}$ incubation, as anticipated, low-intensity emissions of NR-micelles and anti-NR-micelles were observed from cytosol. This is probably due to the micelles started to enter the cells via the endocytosis pathway. In addition, in the case of the anti-NR-micelle added sample, almost no red emission of the anti-NR-micelles from the cell membrane was also observed at $30 \mathrm{~min}$ incubation. This result is probably due to the antibody-antigen recognition of the anti-NR-micelles and the TRPV1 channel on the cell surface is still not interacted in this status. After $3 \mathrm{~h}$, in the case of NRmicelles, red emission mainly occurred inside the cells after $3 \mathrm{~h}$ incubation with NR-micelles, although a low-intensity red emission of NR-micelles was observed on the cell membrane. These results indicate that the NR-micelles mainly entered the cells via endocytosis or were washed away by rinsing before observing the fluorescence. Therefore, most NR-micelles did not remain on the cell surface. In contrast, in the case of the anti-NR-micelles, a strong emission of the anti-NR-micelles was observed on the cell membrane after $3 \mathrm{~h}$ of incubation (yellow arrows in the merged image of the bottom row). This
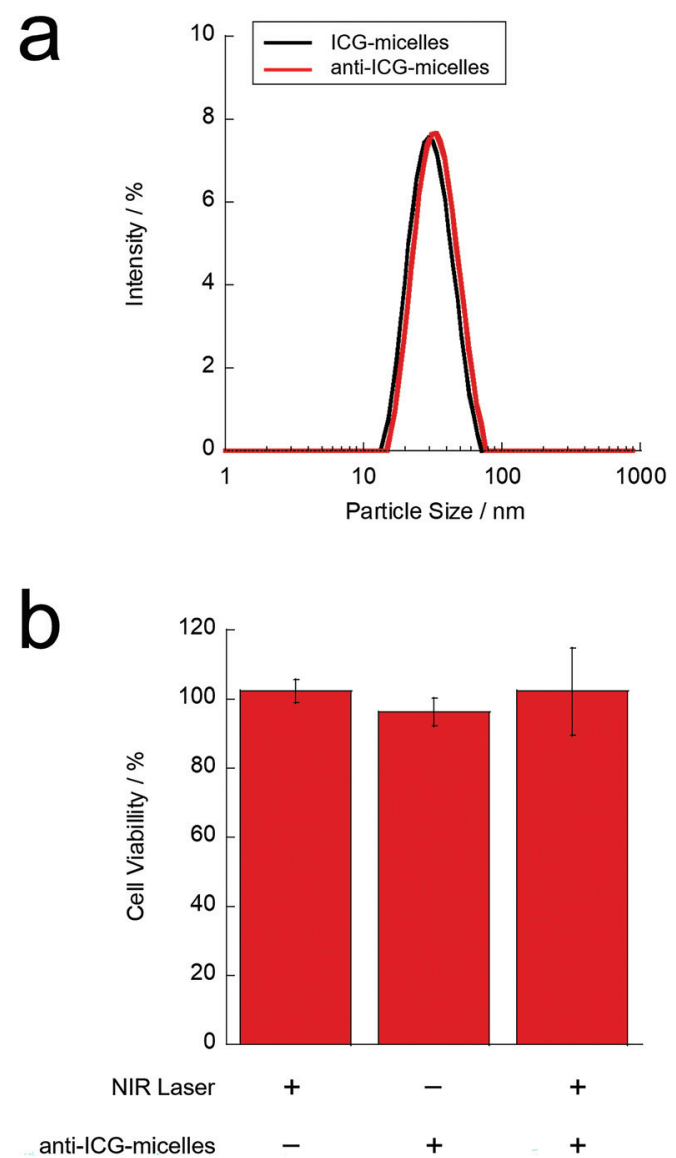

Fig. 5 Characterization of the anti-ICG-micelles. (a) Particle size distributions of the ICG-micelles (black line) and anti-ICG-micelles (red line) in ultrapure water, as measured by DLS. (b) NIR-induced photothermal effect against ND7/23 cells. Cytotoxicity was evaluated by performing WST assays. The data are presented as the mean \pm SD $(n=4)$. 
visual result indicates that a large number of the anti-NRmicelles were probably located on the cell membrane. This result likely reflects the conjugation of the antibody between the micelle surface and the TRPV1 channel on the cell surface. Thus, the antibody-labelled micelles are promising candidates as specific tools for manipulating TRPV1.

To study the NIR light-induced activation of the TRPV1 channel on neuronal cells, anti-TRPV1 antibody-labelled ICG- micelles (anti-ICG-micelles) were prepared via almost the same method used to prepare anti-NR-micelles, as described above. The particle size of the anti-ICG-micelles was characterised by DLS (Fig. 5(a)). Our results showed that, after antibody conjugation, the hydrodynamic diameter of the micelles increased slightly from approximately $35 \mathrm{~nm}$ (ICG-micelles) to approximately $40 \mathrm{~nm}$ (anti-ICG-micelles). This difference likely reflects antibody conjugation on the micelle surface. Furthermore,
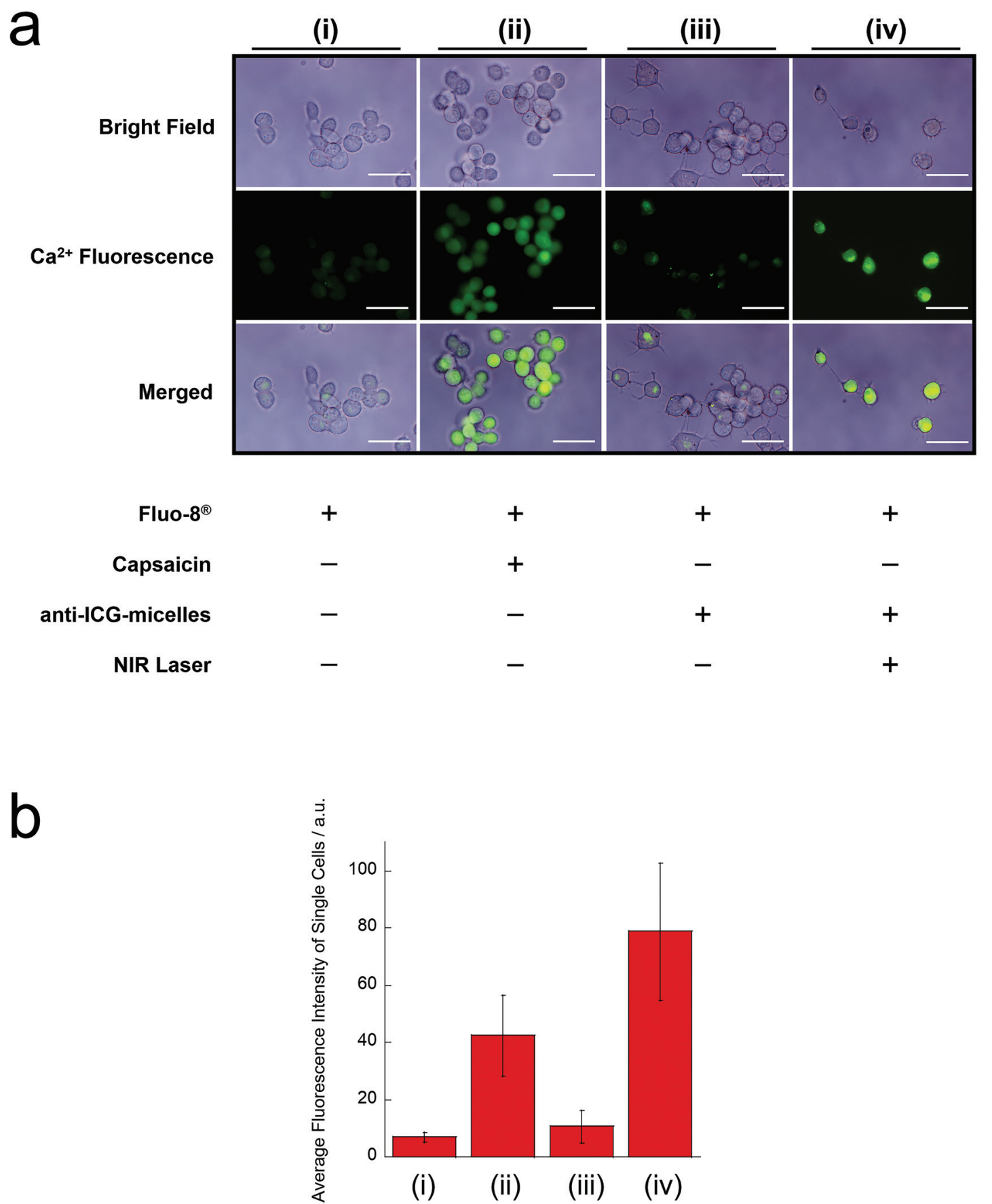

Fig. 6 NIR light-induced activation of ND7/23 cells. (a) Fluorescence microscopy images of ND7/23 cells incubated with ICG-micelle samples and Fluo-8®. (b) Numeric analysis of the average fluorescence intensity of Fluo- $8 \AA$ in the treated single cells. The data are presented as the mean \pm SD $(n=3)$. Scale bar $=50 \mu \mathrm{m}$. 
antibody conjugation on the micelle surface was confirmed by performing Micro BCA assays. The labelling efficiency ( 73\%) of the antibody on the micelle surface was also calculated from the Micro BCA assay results.

Furthermore, the phototoxicity of the anti-ICG-micelles against neurons after NIR irradiation was evaluated by performing WST assays (Fig. 5(b)). The NIR light-induced photothermal effect is used for cancer therapy where an elevated temperature causes cancer cell death. ${ }^{35}$ Therefore, appropriate laser strength and irradiation time are required for safe activation of the TRPV1 channel. Fig. 5(b) shows the WST assay results obtained after treating ND7/23 cells with anti-ICGmicelles and subsequent NIR irradiation. The micelle concentration, laser strength, and irradiation time were $50 \mu \mathrm{g} \mathrm{mL}$ (on ICG), $1 \mathrm{~W} \mathrm{~cm}^{-2}$, and $3 \mathrm{~min}$, respectively. Treatment with NIR laser irradiation alone showed almost no phototoxicity. In addition, the use of anti-ICG-micelles without NIR laser irradiation showed almost no cytotoxicity under the conditions. These results indicate that NIR light irradiation did not exhibit phototoxicity and that the prepared anti-ICGmicelles did not exhibit cytotoxicity. Moreover, the anti-ICGmicelles did not show obvious cytotoxicity after NIR laser irradiation. As shown in Fig. 2(g), the ICG-micelle samples showed an NIR light induced photothermal temperature increase in the cuvette with magnetic stirring at same micelle concentration, laser strength, and irradiation time of cell experiment. However, in the case of cell experiment (Fig. 5(b)), before NIR irradiation, the ICG-micelle added cells were washed with PBS and the unconjugated ICG-micelles were removed from the cells. Thus, the ICG concentration of the cell experiment samples were probably reduced as compared to the temperature measurement samples in the cuvette. In addition, because of the adhered cell samples couldn't stir with stirring bars, only the local region of each ICG-micelles was heated and thus, the temperature increase of culture media was restricted. These results suggest that the photothermal effect of the anti-ICG-micelles does not cause serious cell cytotoxicity under these conditions, and it can be used for the local region heating of the TRPV1 channel. Thus, further TRPV1-activation experiments of neuronal cells were carried out using these conditions.

Finally, we investigated the NIR light-induced remote activation of the TRPV1 channel in neuronal cells (Fig. 6). TRPV1 activation on the cell membranes of neurons was evaluated using TRPV1 (+) ND7/23 cells. The influx of $\mathrm{Ca}^{2+}$ into the cells was visualised using the $\mathrm{Ca}^{2+}$ indicator Fluo-8® (Fig. 6(a)). Fig. 6(b) shows the average fluorescence intensities of single cells from Fig. 6(a), expressed in numerical terms. As a positive control, because TRPV1 is known as the capsaicin receptor, ND7/23 cells were treated with capsaicin, and Fluo-8® emission was observed. We observed an obvious increase in the green Fluo-8® emission after capsaicin treatment (Fig. 6(a)(ii)), when compared with the untreated sample (Fig. 6(a)(i)) and micelles without NIR irradiation (Fig. 6(a)(iii)). These results indicate that the TRPV1 channel of ND7/23 cells was activated by capsaicin and that the $\mathrm{Ca}^{2+}$ ion influx occurred.
Furthermore, the strong Fluo-8® emission was confirmed after incubation with anti-ICG-micelles and NIR light irradiation (Fig. 6(a)(iv)). This result strongly supports the interpretation that the TRPV1 channel was activated by the photothermal effect of the anti-ICG-micelles under NIR light irradiation, which was followed by a robust $\mathrm{Ca}^{2+}$ influx. In addition, converting the results to a numerical form also support the obtained results (Fig. 6(b)). Based on these results, we anticipate that the anti-ICG-micelles will be suitable for use as remote-activation tools for TRPV1 channels in neuronal cells.

\section{Conclusions}

In this study, we developed a biodegradable material-based remote-activation method for the temperature-sensitive channel TRPV1 expressed on neurons. Biodegradable/photothermal polymer micelles (ICG-micelles) were successfully prepared via simple one-pod micelle formation. The obtained nanoparticles were well dispersed and showed excellent biodegradability and biocompatibility. Furthermore, the ICGmicelles also showed excellent photothermal effects under tissue-penetrable NIR irradiation. Additionally, the ICGmicelles exhibited strong NIR emission under NIR excitation. Therefore, the ICG-micelles can also be used for deep-tissue in vivo fluorescence imaging, indicating that they are promising nanomaterials for in vivo deep-tissue manipulations and observations. Moreover, NIR light-triggered remote activation of neurons was successfully performed. Specific conjugation of the ICG-micelles with the TRPV1 channel on the cell membrane was achieved by decoration of the micelle surface with anti-TRPV1 antibodies, which accelerated the $\mathrm{Ca}^{2+}$ ion influx into neuronal cells and successfully induced by NIR irradiation. Thus, the biodegradable/photothermal polymer micelles developed here can be applied for the noninvasive remote activation of neuron cells.

\section{Author contributions}

M. Kamimura was the main project leader and conceived the overall research project. W. Chen and T. Onoe performed the experiments and collected the data. The manuscript was originally drafted by W. Chen and M. Kamimura. All authors read and approved the final manuscript prior to submission.

\section{Conflicts of interest}

There are no conflicts to declare.

\section{Acknowledgements}

This research was supported by a Grant-in-Aid for Scientific Research (B) (grant number 18H03538) from the Japan Society for the Promotion of Science. 


\section{Notes and references}

1 J. Rivnay, H. Wang, L. Fenno, K. Deisseroth and G. G. Malliaras, Sci. Adv., 2017, 3, e1601649.

2 J. Li, H. Duan and K. Pu, Adv. Mater., 2019, 31, 1901607.

3 B. Özkale, M. S. Sakar and D. J. Mooney, Biomaterials, 2021, 267, 120497.

4 J. Dobson, Nat. Nanotechnol., 2008, 3, 139.

5 S. A. Stanley, J. Sauer, R. S. Kane, J. S. Dordick and J. M. Friedman, Nat. Med., 2015, 21, 92.

6 A. Tay and D. Di Carlo, Nano Lett., 2017, 17, 886.

7 J. Moon, M. G. Christiansen, S. Rao, C. Marcus, D. C. Bono, D. Rosenfeld, D. Gregurec, G. Varnavides, P.-H. Chiang, S. Park and P. Anikeeva, Adv. Funct. Mater., 2020, 30, 2000577.

8 J.-u. Lee, W. Shin, Y. Lim, J. Kim, W. R. Kim, H. Kim, J.-H. Lee and J. Cheon, Nat. Mater., 2021, 20, 1029.

9 Z. Qiu, J. Guo, S. Kala, J. Zhu, Q. Xian, W. Qiu, G. Li, T. Zhu, L. Meng, R. Zhang, H. C. Chan, H. Zheng and L. Sun, iScience, 2019, 21, 448.

10 Z. Qiu, S. Kala, J. Guo, Q. Xian, J. Zhu, T. Zhu, X. Hou, K. F. Wong, M. Yang, H. Wang and L. Sun, Cell Rep., 2020, 32, 108033.

11 S. A. Chechetka, M. Doi, B. P. Pichon, S. Bégin-Colin and E. Miyako, Nanotechnology, 2016, 27, 475102.

12 Y. Yu, X. Yang, S. Reghu, S. C. Kaul, R. Wadhwa and E. Miyako, Nat. Commun., 2020, 11, 4117.

13 A.-P. Koivisto, M. G. Belvisi, R. Gaudet and A. Szallasi, Nat. Rev. Drug Discovery, 2022, 21, 41.

14 P. Stokłosa, A. Borgström, S. Kappel and C. Peinelt, Int. J. Mol. Sci., 2020, 21, 1877.

15 H. Huang, S. Delikanli, H. Zeng, D. M. Ferkey and A. Pralle, Nat. Nanotechnol., 2010, 5, 602.

16 R. Chen, G. Romero, M. G. Christiansen, A. Mohr and P. Anikeeva, Science, 2015, 347, 1477.

17 H. Nakatsuji, T. Numata, N. Morone, S. Kaneko, Y. Mori, H. Imahori and T. Murakami, Angew. Chem., Int. Ed., 2015, 54, 11725.

18 Y. Lyu, C. Xie, S. A. Chechetka, E. Miyako and K. Pu, J. Am. Chem. Soc., 2016, 138, 9049.

19 R. Munshi, S. M. Qadri, Q. Zhang, I. Castellanos Rubio, P. del Pino and A. Pralle, eLife, 2017, 6, e27069.

20 X. Zhen, C. Xie, Y. Jiang, X. Ai, B. Xing and K. Pu, Nano Lett., 2018, 18, 1498.

21 W.-T. Chen, G.-B. Lin, S.-H. Lin, C.-H. Lu, C.-H. Hsieh, B.-L. Ma and C.-Y. Chao, PLoS One, 2018, 13, e0191078.

22 W. Gao, Y. Sun, M. Cai, Y. Zhao, W. Cao, Z. Liu, G. Cui and B. Tang, Nat. Commun., 2018, 9, 231.

23 Z. Ma, J. Zhang, W. Zhang, M. F. Foda, Y. Zhang, L. Ge and H. Han, iScience, 2020, 23, 101049.

24 K. Deisseroth, Nat. Methods, 2011, 8, 26.

25 O. Yizhar, L. E. Fenno, T. J. Davidson, M. Mogri and K. Deisseroth, Neuron, 2011, 71, 9.
26 L. Fenno, O. Yizhar and K. Deisseroth, Annu. Rev. Neurosci., 2011, 34, 389.

27 S. Shah, J.-J. Liu, N. Pasquale, J. Lai, H. McGowan, Z. P. Pang and K.-B. Lee, Nanoscale, 2015, 7, 16571.

28 S. Chen, A. Z. Weitemier, X. Zeng, L. He, X. Wang, Y. Tao, A. J. Y. Huang, Y. Hashimotodani, M. Kano, H. Iwasaki, L. K. Parajuli, S. Okabe, D. B. L. Teh, A. H. All, I. TsutsuiKimura, K. F. Tanaka, X. Liu and T. J. McHugh, Science, 2018, 359, 679.

29 A. H. All, X. Zeng, D. B. L. Teh, Z. Yi, A. Prasad, T. Ishizuka, N. Thakor, Y. Hiromu and X. Liu, Adv. Mater., 2019, 31, 1803474.

30 A. M. Smith, M. C. Mancini and S. Nie, Nat. Nanotechnol., 2009, 4, 710.

31 M. J. Caterina, M. A. Schumacher, M. Tominaga, T. A. Rosen, J. D. Levine and D. Julius, Nature, 1997, 389, 816.

32 E. Miyako, J. Russier, M. Mauro, C. Cebrian, H. Yawo, C. Ménard-Moyon, J. A. Hutchison, M. Yudasaka, S. Iijima, L. De Cola and A. Bianco, Angew. Chem., Int. Ed., 2014, 53, 13121.

33 S. P. Sanchez-Rodriguez, J. P. Sauer, S. A. Stanley, X. Qian, A. Gottesdiener, J. M. Friedman and J. S. Dordick, Biotechnol. Bioeng., 2016, 113, 2228.

34 J. Song, J.-B. Pan, W. Zhao, H.-Y. Chen and J.-J. Xu, Chem. Commun., 2020, 56, 6118.

35 D. Jaque, L. Martínez Maestro, B. del Rosal, P. HaroGonzalez, A. Benayas, J. L. Plaza, E. Martín Rodríguez and J. García Solé, Nanoscale, 2014, 6, 9494.

36 B. E. Schaafsma, J. S. D. Mieog, M. Hutteman, J. R. van der Vorst, P. J. K. Kuppen, C. W. G. M. Löwik, J. V. Frangioni, C. J. H. van de Velde and A. L. Vahrmeijer, J. Surg. Oncol., 2011, 104, 323.

37 X. Zheng, D. Xing, F. Zhou, B. Wu and W. R. Chen, Mol. Pharm., 2011, 8, 447.

38 F. Yan, H. Wu, H. Liu, Z. Deng, H. Liu, W. Duan, X. Liu and H. Zheng, J. Controlled Release, 2016, 224, 217.

39 M. V. Marshall, J. C. Rasmussen, I. C. Tan, M. B. Aldrich, K. E. Adams, X. Wang, C. E. Fife, E. A. Maus, L. A. Smith and E. M. Sevick-Muraca, Open Surg. Oncol. J., 2010, 2, 12.

40 R. Bhavane, Z. Starosolski, I. Stupin, K. B. Ghaghada and A. Annapragada, Sci. Rep., 2018, 8, 14455.

41 H. Cabral, K. Miyata, K. Osada and K. Kataoka, Chem. Rev., 2018, 118, 6844.

42 D. Hwang, J. D. Ramsey and A. V. Kabanov, Adv. Drug Delivery Rev., 2020, 156, 80.

43 Y. Geng and D. E. Discher, J. Am. Chem. Soc., 2005, 127, 12780.

44 H. Cho and G. S. Kwon, ACS Nano, 2011, 5, 8721.

45 H. Cho, T. C. Lai and G. S. Kwon, J. Controlled Release, 2013, 166, 1.

46 M.-H. Chan, Y.-T. Pan, I.-J. Lee, C.-W. Chen, Y.-C. Chan, M. Hsiao, F. Wang, L. Sun, X. Chen and R.-S. Liu, Small, 2017, 13, 1700038. 